\title{
Systematic modeling of microfluidic concentration gradient generators
}

\author{
Yi Wang ${ }^{1}$, Tamal Mukherjee ${ }^{2}$ and Qiao Lin $^{3}$ \\ ${ }^{1}$ CFD Research Corporation, Huntsville, AL 35805, USA \\ ${ }^{2}$ Department of Electrical and Computer Engineering, Carnegie Mellon University, \\ PA 15213, USA \\ ${ }^{3}$ Department of Mechanical Engineering, Columbia University, New York 10027, \\ USA \\ E-mail: q12134@columbia.edu
}

Received 10 May 2006, in final form 14 July 2006

Published 1 September 2006

Online at stacks.iop.org/JMM/16/2128

\begin{abstract}
This paper presents a systematic modeling methodology for microfluidic concentration gradient generators. The generator is decomposed into a system of microfluidic elements with relatively simple geometries.

Parameterized models for such elements are analytically developed and hold for general sample concentration profiles and arbitrary flow ratios at the element inlet; hence, they are valid for concentration gradient generators that rely on either complete or partial mixing. The element models are then linked through an appropriate set of parameters embedded at the element interfaces. This yields a systematic, lumped-parameter representation of the entire generator in terms of a network of gradient-generation elements. The system model is verified by numerical analysis and experimental data and accurately captures the overall effects of network topologies, element sizes, flow rates and reservoir sample concentrations on the generation of sample concentration gradient. Finally, this modeling methodology is applied to propose a novel and compact microfluidic device that is able to create concentration gradients of complex shapes by juxtaposing simple constituent profiles along the channel width.
\end{abstract}

(Some figures in this article are in colour only in the electronic version)

\section{Introduction}

Concentration gradients and concentration arrays of diffusible samples play important roles in the study of cell biology (e.g., chemotaxis [1, 2]), biochemistry [3, 4], surface patterning and microfabrication $[5,6]$. Conventionally, the Boyden chamber [7], pipette [8], gel [9] or their derivatives [10, 11] are mainly used to release the sample and investigate cell behavior subjected to the concentration gradient. However, these techniques are not effective in generating spatially stable gradients of complex shapes due to the unbalanced sample flux into and from the region of interest [12]. Therefore, a technique to generate and maintain predictable complex gradients of the sample concentration over a long period of time that can be employed to examine the correlation between sample gradients and cell response [12] is strongly desired. In addition, development of sample concentration arrays also enables the high-throughout assays (e.g., immunoassay and enzyme assay) and efficient multi-dimensional screens for combinational chemistry [3, 4, 13].

Recently, laminar diffusion-based microfluidic networks have been extensively studied for the concentrationrelated analysis since they allow accurate and reproducible manipulation of the locations and quantities of samples released into the system [12]. In general, these devices can be classified into two categories: complete mixing- and partial mixing-based. Complete mixing devices generally involve serially recombining, mixing and splitting the sample solution and buffer (or sample with different concentration). Sample mixing before each splitting needs to be effectively complete (uniform concentration along the channel width) to allow use of models that represent channels and sample flow rates as resistors and electrical currents in an electrical analogy. 
Jacobson et al [14] proposed a serial mixing network driven by electrokinetic flow to achieve a continuously diluted sample concentration array. This approach later was used in pressure driven flow [3] to perform high-throughput fluorescent immunoassays and quantitatively analyze multiple antibodies in parallel. Dertinger et al [12] and Jeon et al [15] proposed a tree-like microfluidic network to generate concentration gradients of complex shapes (e.g., linear, parabolic and periodic) along the channel width. Sample solutions with different concentrations were supplied at multiple inlets, then mixed, split and recombined in branch channels, which eventually converged to the main channel to yield a transverse complex profile (i.e., across the channel width). This device was later improved by Lin et al [16], who integrated a simple 'mixer module' with the gradient generating network to enable on-chip dilution of initial samples and obtain dynamically controlled temporal and spatial gradients. More recently, Walker et al [17] have studied the effects of flow and diffusion on chemotaxis in such devices using a simplified numerical model as well as experiments. Nevertheless, to ensure the transversely complete sample mixing before each splitting, such gradient generators [12, 15-17] often use either bulky network configurations that are prone to leakage and clogging, or chaotic advective mixers [5] that are laborious in layout and fabrication. Additionally, profiles produced at the output channel are also discontinuous.

Concentration gradient or array generators fully relying on diffusion-based partial mixing are simple in design and fabrication. Holden et al [13] reported a Y-shape laminar microfluidic diffusion diluter (mDD) that took advantage of lateral mixing of different samples to generate transverse concentration gradients. An analytical model was also developed to accurately predict the output profiles. Walker et al [18] designed a cross-mixing microfluidic device that created a transverse bell-shaped concentration profile of a virus to study the cell infection within a microscale environment. Biddiss et al [19] presented a method to generate linear and bell-shaped concentration gradients in electrokinetic flow, which can be varied by adjusting the applied voltage and charge heterogeneity of the channel surface. While these devices are effective in generating relatively simple (e.g., approximately linear or bell shape) concentration gradients, they have not been adequately studied for generating complex (such as sawtooth or bell-shape in $[2,12])$ profiles. This is, perhaps, primarily caused by a lack of efficient and accurate models to accurately analyze the variation of sample concentration profiles in the network.

To address this issue, this paper proposes a systematic modeling approach for efficient design of both complete and partial mixing-based concentration gradient generators. In our approach, a complex generator is geometrically decomposed into a set of simple elements. Analytical models for individual elements are derived to accurately capture the dependence of fluid and sample transport on device topologies, element sizes, material properties and initial reservoir sample concentrations. Proper parameters are embedded at input and output terminals (i.e., inlets and outlets) of these element models to pass fluidic and concentration information between adjacent elements. As a result, the network of gradient generators can be represented as a system model by linking the individual elements [20].
The modeling results are validated using experimental data extracted from the literature [17] as well as full numerical analysis. To demonstrate its utility, this approach is used to propose a novel and compact microfluidic device that is capable of generating transverse complex (e.g., saw-toothshaped, multiple-bell-shaped) concentration profiles.

Compared to previous studies, our effort exhibits three significant novelties. First, in contrast to the previous work focusing on electrokinetic flow [20, 21], this paper extends the element models to accommodate pressure-driven flow. Second, while resistor-based models [12, 14], which exploit the analogy between electric circuitry and fluidsample transport, impose the complete mixing constraint on device design, our approach allows concentration profiles (rather than a single average concentration value) to propagate within the entire network. Therefore, it not only yields all information attainable by resistor-based models, but also captures spatial variations of the sample gradient generation as well as its dependence on design parameters [13, 17]. Third, we juxtapose simple constituent profiles resulting from partial mixing $[13,18]$ to construct complex gradients. This obviates complicated device features normally required by complete mixing, such as long zig-zag mixing channels, chaotic advective mixers and bulky network structures $[5,12,15]$. Therefore, our design affords simple layout and fabrication, improved reliability, and enhanced performance (e.g., smooth concentration profiles).

The paper is organized as follows. The systematic modeling approach is first briefly introduced in section 2, which is followed by analytical modeling of fluidic and sample transport in individual elements, model validity, and system integration (section 3). The system model is then validated using experimental data and numerical analysis (section 4). In section 5 , the model is applied to propose a novel partial mixing-based generator and is verified by numerical analysis.

\section{Systematic representation of gradient generators}

Our systematic approach can be illustrated with a tree-like gradient generator that consists of reservoirs, channels, and junctions (figure $1(a)$ ) and produces linear concentration profiles $[2,17]$. The sample and buffer are released from their individual reservoirs, mixed as they traverse the device, and finally enter the outlet. We represent the device as a collection of interconnected elements (figure 1(b)), including mixing channels, combiners (each with two input and one output streams), splitters (each with one input and two output streams) and reservoirs. Note that the multi-input junction at the inlet of the main channel is modeled as a cascade concatenation of combiners. Then a system model can be constructed from the element models, which are linked in correspondence to the device topology. An appropriate set of parameters (called interface parameters) is embedded at the element interfaces to enable communication of flow and concentration information between adjacent elements. As all element models are parameterized and reusable, the user can quickly compose and modify the device design in an efficient top-down manner [20, 21]. 


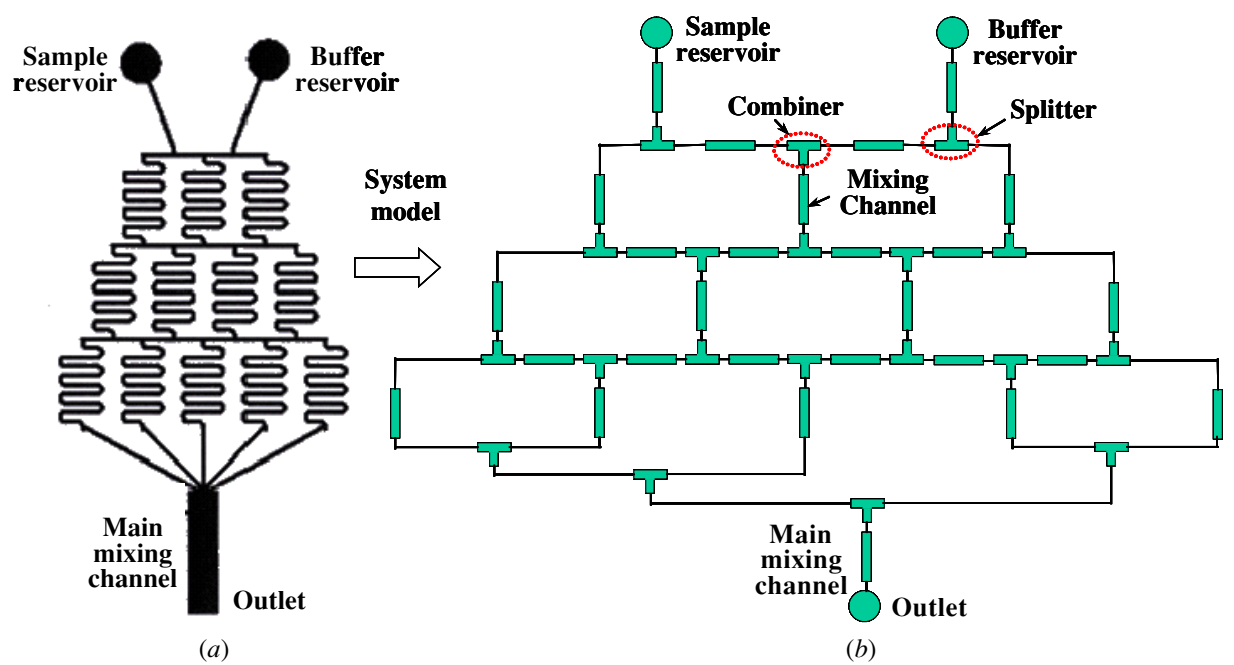

Figure 1. (a) Sketch of a tree-like gradient generator. (b) Schematic for the systematic representation of the generator.

\section{Modeling of concentration gradient generators}

In this section, we will first present models for the constituent elements in our systematic representation scheme. These element models will then be integrated for systematic evaluation of generator designs.

\subsection{Element models}

We develop models for mixing channels, combiners and splitters, which are characterized by pressure-driven laminar flow and molecular diffusion-based sample transport.

3.1.1. Mixing channels. In this section, we consider analytical solutions of pressure-driven fluid flow and sample transport in a mixing channel. Neglecting entry regions at the channel inlet, the fluid flow is fully developed within the channel and governed by

$$
\frac{\partial^{2} u}{\partial y^{2}}+\frac{\partial^{2} u}{\partial z^{2}}=-\frac{1}{\mu} \frac{\partial P}{\partial x}
$$

where $x, y$ and $z$ are axial, transverse and depth-wise coordinates respectively, $\mu$ is the buffer's dynamic viscosity, $u$ is the axial fluid velocity. Here, $x \in[0, L], y \in[0, w]$ and $z \in[0, h]$; and $L, w$ and $h$ are the channel's length, width and depth, respectively. For fully developed flow, $\mathrm{d} P / \mathrm{d} x=$ $\Delta P / L=$ const, where $\Delta P$ is the pressure difference applied over the channel length. Equation (1) can be solved analytically [22] to yield the flow resistance of the channel:

$$
R=\frac{\Delta P}{q}=\frac{12 \beta L \mu}{w^{4}\left[1-\frac{192 \beta}{\pi^{5}} \sum_{i=1,3,5, \ldots}^{\infty} \frac{\tanh (i \pi / 2 \beta)}{i^{5}}\right]}
$$

where $q=\int_{0}^{h} \int_{0}^{w} u \mathrm{~d} y \mathrm{~d} z$ is the volumetric buffer flow rate, and $\beta=w / h$ is the channel's aspect ratio. Equation (2) shows that in terms of fluid flow each mixing channel can be represented as a resistor. Thus, a steady-state pressure-driven flow network can be treated as a network of electrical resistors, with pressures and volumetric flow rates analogous to voltages and currents. Kirchhoff's and Ohm's laws hence can be used to compute pressures at element terminals and flow rates through the elements.
Steady-state sample transport in a mixing channel is governed by the convection-diffusion equation

$$
u \frac{\partial c}{\partial x}=D\left(\frac{\partial^{2} c}{\partial x^{2}}+\frac{\partial^{2} c}{\partial y^{2}}+\frac{\partial^{2} c}{\partial z^{2}}\right)
$$

where $c$ is the sample concentration and $D$ is the sample's molecular diffusivity. Two assumptions are made to simplify this equation. First, we assume that the channel is flat, i.e., the aspect ratio is large: $\beta \gg 1$. It can then be shown [13, 23, $24]$ that for flat channels velocity profiles along the channel width are approximately uniform and sample transport is not affected by the depth-wise velocity distribution due to small diffusion times along the depth. This implies that the axial velocity $u$ in equation (3) can be replaced with the crosssectional average velocity, $U=q / w h$. In addition, depth-wise concentration variations can be ignored, i.e., $\partial^{2} c / \partial z^{2} \approx 0$. The second assumption is that the channel is long, i.e., $h \ll L$ and $w \ll L$, which implies that axial diffusion is also negligible (i.e., $\partial^{2} c / \partial x^{2} \ll \partial^{2} c / \partial y^{2}$ ). Thus, equation (3) is simplified to

$$
U \frac{\partial c}{\partial x}=D \frac{\partial^{2} c}{\partial y^{2}}
$$

The sample concentration profiles at the inlet and outlet can be represented in terms of Fourier series: $c_{\text {in }}(\eta)=$ $\sum_{n=0}^{\infty} d_{n}^{\text {(in) }} \cos (n \pi \eta)$ and $c_{\text {out }}(\eta)=\sum_{n=0}^{\infty} d_{n}^{\text {(out) }} \cos (n \pi \eta)$, where $\eta=y / w$ is the normalized transverse coordinate along the channel width. Equation (4) can be solved, also in Fourier series, to yield the input-output relationship of the Fourier coefficients $d_{n}$

$$
d_{n}^{\text {(out) }}=d_{n}^{\text {(in) }} \mathrm{e}^{-(n \pi)^{2} \tau}
$$

where $\tau=L /(w P e)$ is the dimensionless sample residence time in the channel, $P e=U w / D$ is the Peclet number, which is a characteristic ratio of convective and diffusive rates in sample transport. Note that $d_{0}^{\text {(in) }}=d_{0}^{\text {(out) }}$ is the average concentration over the channel cross section. In contrast to results obtained in [13], equation (5) holds for arbitrary flow rates and general concentration profiles at the inlet.

Equation (5) is also applicable to a curve-shaped turn of rectangular cross section with the centerline arc length given by $L=r_{\mathrm{c}} \varphi$, where $r_{\mathrm{c}}$ is the mean radius and $\varphi$ is the 


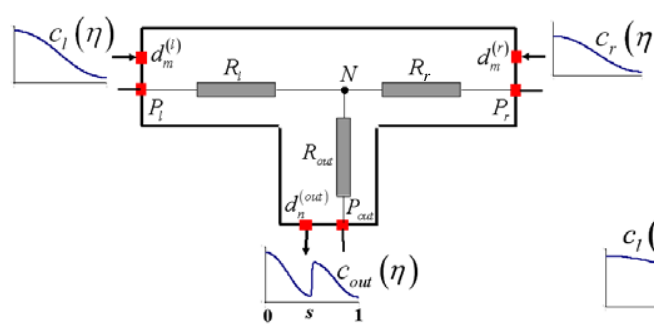

(a)

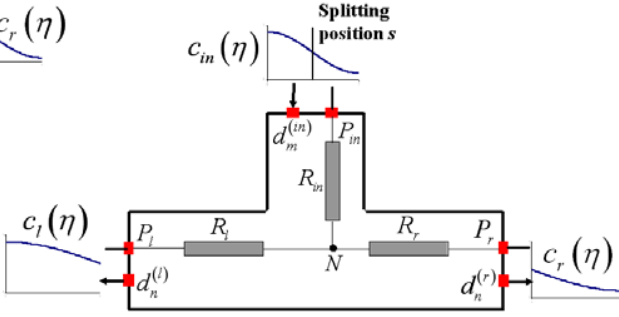

(b)

Figure 2. Modeling approach for the $(a)$ combiner and $(b)$ splitter. $s$ is the merging or splitting position between the two branch streams.

included angle of the turn. It should be pointed out that for electrokinetic flow, as long as the similitude between electric fields and electrokinetic flow holds [20], equations (4) and (5) are valid regardless of the channel's aspect ratio.

3.1.2. Combiners. The combiner is another element commonly used in gradient generators. In a combiner two incoming streams with certain sample concentration profiles are juxtaposed and emerge as a single combined stream. As the flow path lengths of the combiner are negligible, such an element can be assumed to have zero physical size. Thus, a combiner can be represented as three resistors with zero fluidic resistance between each terminal and the internal node $N$ (figure 2(a)):

$$
R_{1}=R_{\mathrm{r}}=R_{\text {out }}=0
$$

where the subscripts $1, \mathrm{r}$ and out represent the left and right inlets and the outlet, respectively.

Denote by $d_{m}^{(\mathrm{l})}$ and $d_{m}^{(\mathrm{r})}(m=0,1,2, \ldots)$ the Fourier coefficients of the sample concentration profiles at the left and right inlets, respectively. They can be used to obtain the Fourier coefficients $d_{n}^{\text {(out) }}(n=0,1,2, \ldots)$ at the combiner outlet as follows $[20,21]$ with an assumption that sample and buffer have same viscosity,

$$
d_{n}^{\text {(out) }}=\left\{\begin{array}{l}
d_{0}^{(\mathrm{l})} s+d_{0}^{(\mathrm{r})}(1-s), \quad \text { if } n=0 \\
s \sum_{m=0}^{\infty, \text { if } m \neq n s} d_{m}^{(\mathrm{l})} \frac{f_{1} \sin \left(f_{2}\right)+f_{2} \sin \left(f_{1}\right)}{f_{1} f_{2}}+s \sum_{m=0}^{\infty, \text { if } m=n s} \\
\quad \times d_{m}^{(\mathrm{l})}+(1-s) \sum_{m=0}^{\infty, \text { if } m=n(1-s)}(-1)^{n-m} d_{m}^{(\mathrm{r})} \\
+2(-1)^{n}(1-s) \sum_{m=0}^{\infty, \text { if } m \neq n(1-s)} d_{m}^{(\mathrm{r})} \\
\times\left(\frac{\cos \left(F_{2} / 2\right) \sin \left(F_{1} / 2\right)}{F_{1}}+\frac{\cos \left(F_{1} / 2\right) \sin \left(F_{2} / 2\right)}{F_{2}}\right), \\
\text { if } n \geqslant 1
\end{array}\right.
$$

where $s=q_{1} /\left(q_{1}+q_{\mathrm{r}}\right)$ is the flow ratio, the normalized flow rate of the left-side stream, which gives the normalized position of the interface between the incoming streams. In equation (7), $f_{1}=(m-n s) \pi, f_{2}=(m+n s) \pi, F_{1}=(m+n-n s) \pi$ and $F_{2}=(m-n+n s) \pi$. Since the sample concentration profiles from the inlets are scaled down at the outlet, the Fourier series components at the inlet are not orthogonal to those at the outlet. Therefore, different indices $m$ and $n$ are used at the inlets and outlet, respectively.

3.1.3. Splitters. The splitter is the dual of the combiner, and splits a single incoming stream into two that emerge at the leftand right-side outlets (figure $2(b)$ ). Such an element can also be represented by three zero-resistance flow resistors,

$$
R_{\text {in }}=R_{1}=R_{\mathrm{r}}=0
$$

where subscripts in, 1 and $r$ represent the inlet, the left and right outlets, respectively.

Let $d_{m}^{(\text {in) }}(m=0,1,2, \ldots)$ be the Fourier coefficients of the sample concentration profile at the inlet. The Fourier coefficients at the left and right outlets are given by

$$
d_{n}^{(\mathrm{l})}=\left\{\begin{array}{c}
d_{0}^{(\text {in) }}+\sum_{m=1}^{\infty} d_{m}^{(\text {in })} \sin \left(\phi_{1}\right) / \phi_{1}, \quad \text { if } n=0 \\
2 \sum_{m=0}^{\infty, \text { if } m \neq n / s} d_{m}^{\text {(in) }}(-1)^{n+1} \phi_{1} \sin \left(\phi_{1}\right) / f_{1} f_{2} \\
\quad+\sum_{m=0}^{\infty, \text { if } m=n / s} d_{m}^{\text {(in) }}, \quad \text { if } \quad n \geqslant 1
\end{array}\right.
$$

and

$$
d_{n}^{(\mathrm{r})}=\left\{\begin{array}{l}
d_{0}^{(\mathrm{in})}-\sum_{m=1}^{\infty} d_{m}^{(\mathrm{in})} \sin \left(\phi_{1}\right) / \phi_{2}, \quad \text { if } n=0 \\
2 \sum_{m=0}^{\infty, \text { if } m \neq n /(1-s)} d_{m}^{(\text {in })} \phi_{2} \sin \left(\phi_{1}\right) / F_{1} F_{2} \\
\quad+\sum_{m=0}^{\infty, \text { if } m=n /(1-s)}(-1)^{m-n} d_{m}^{(\text {in })}, \quad \text { if } n \geqslant 1
\end{array}\right.
$$

where $f_{1}=(n-m s) \pi, f_{2}=(n+m s) \pi, F_{1}=(n+m-$ $m s) \pi, F_{2}=(n-m+m s) \pi, \phi_{1}=m s \pi$ and $\phi_{2}=m(1-$ $s) \pi$. Here, $s=q_{1} /\left(q_{1}+q_{\mathrm{r}}\right)$ is the splitting flow ratio, as well as the normalized interface position between the two split streams. Again, in equations (9) and (10), the sample and buffer streams are assumed to have equal viscosity. By the same reason, different indices $m$ and $n$ are used at the inlet and outlets.

\subsection{Validity of element models}

The element models are valid when fluid streams in an element are laminar and when axial diffusion effects are negligible compared with transverse diffusion effects. In addition, secondary flow in junctions (combiners and splitters) and turns must be negligible. These impose constraints to operational Reynolds, Dean and Peclet numbers in our models, which are in general satisfied in practical gradient generators.

First, for the flow to be laminar in closed shapes such as channels and junctions, the Reynolds number in general needs to be much smaller than 2300 [22]. In addition, secondary flows (which may be chaotic) in junctions such as combiners and splitters must be negligible, which requires the Reynolds number to be further smaller, e.g., $R e \ll 100$ [25]. This is indeed true for concentration gradient/array generators, which critically depends on laminar-diffusion behavior of micro flow. Such devices typically involve flows of several $\mathrm{mm} \mathrm{s}^{-1}$ or smaller in velocity $[2,5,12$, $13,17,18,26]$, considering its physiological relevance [17] and practical requirements on sample consumption, chip size, reaction time, and reproducible, precise and accurate species quantity control. With the microscale dimensions of gradient generation elements, the Reynolds number is typically smaller 
than 1. Thus, our junction models are valid in practice. For example, in all concentration gradient generator examples below (section 5), the maximum flow speed is $1.53 \mathrm{~mm} \mathrm{~s}^{-1}$, yielding a Reynolds number of 0.15 .

We have also extended the model (equations (2) and (5)) to turns with the assumption that curvature-induced secondary flow is negligible. Generally, this assumption is valid when the Dean number $(D e)$, a measure of the relative magnitude of the secondary flow with respect to the main flow, is of 1 or smaller [27-29]. This dimensionless parameter is defined for a turn with a rectangular cross section as $D e=R e \sqrt{d_{\mathrm{h}} / r_{\mathrm{c}}}$ [27-29], where $d_{\mathrm{h}}$ is the channel's hydraulic diameter. For channels with large aspect ratios $(\beta \gg 1)$ as considered by our models, $D e \approx \operatorname{Re} \sqrt{2 b / \beta}$, where we recall that $w$ is the channel width and $b=w / r_{\mathrm{c}}$ is the channel curvature. As $b$ and $R e$ are both smaller than 1 in our proposed generators, we arrive at $D e<1$. Therefore secondary flow induced by channel curvature should be negligible.

Finally, axial diffusion is neglected in the mixing channel model. This is valid when $P e \gg 1$, so that the effect of axial diffusion is negligible compared with that of transverse diffusion. This is generally true for practical concentration generators. Specifically, for such devices, the channel width $w \sim 100 \mu \mathrm{m}$ or larger, which allows good resolution of detection and array output and slow gradient decay. In addition, flow speeds are on the order of $U \sim 1 \mathrm{~mm} \mathrm{~s}^{-1}$ and the sample diffusivity $D \sim 10^{-10} \mathrm{~m}^{2} \mathrm{~s}^{-1}$, yielding $P e \sim 1000$. Therefore, axial diffusion is in general negligible in concentration gradient generators considered by our model.

\subsection{Systematic modeling}

We now integrate element models to obtain a system model for simulation. The challenge is to communicate both fluid and sample transport information between neighboring element models at their interfaces. This is accomplished by defining two sets of interface parameters at inlets and outlets of the element model as shown in figure 2. For an element $j$, we have fluid pressure $\left(P_{i}\right)^{j}$ at its terminals and $\left\{d_{n}^{(i)}\right\}^{j}$, the set of Fourier coefficients of the concentration profile along the channel width [20], where the index $i$ has values in, out, 1 or $\mathrm{r}$, respectively, to represent the element's inlet, outlet, left or right inlets/outlets (for combiners and splitters). Parameters between two neighboring elements are then set equal, i.e., $\left(P_{\text {in }}\right)^{j+1}=\left(P_{\text {out }}\right)^{j}$ and $\left\{d_{n}^{\text {(in) }}\right\}^{j+1}=\left\{d_{n}^{\text {(out) }}\right\}^{j}$ because of continuity requirements.

Simulation using the system model involves computing both fluidic parameters (including pressure and flow rates) and sample concentrations in the network. Given system topologies, element geometries and applied pressures (or equivalently volumetric flow rates) at reservoirs, pressures $\left(P_{i}\right)^{j}$ at the element terminals are first computed for the entire generator network by Kirchhoff's and Ohm's laws based on electric analogy. The flow rate $(q)$, average velocity and direction of the stream within each element, as well as the flow ratio at combiners and splitters are then calculated. With these results and user-input sample diffusivity $D$, Fourier coefficients $\left\{d_{n}^{\text {(out) }}\right\}^{j}$ of sample concentrations at the outlets of element $j$ are determined from the corresponding values at the element's inlets, and then assigned as an input to the inlet of the immediately downstream element $j+1$. This procedure starts from the most upstream sample reservoir.

The system model is implemented using symbolic expression and solved with numerical substitution in Mathematica 5.0 [30]. To capture abrupt gradients such as saw-tooth-shaped profiles, one hundred Fourier terms $(n=99)$ are found to yield sufficient accuracy. For smooth gradients such as multiple-bell-shaped profiles, ten Fourier terms $(n=9)$ are used.

\section{Model validation}

In this section, our system modeling results on concentration gradient generation will be validated numerically and experimentally.

\subsection{Numerical analysis}

Numerical analysis is performed with the commercial finite volume method (FVM) simulation package CFD-ACE+ [31] and used as a baseline for validation of our system models. We invoke the CFD-ACE+ modules of incompressible fluid flow (Navier-Stokes equation) and biochemistry (convectiondiffusion equation without reaction) to solve for the buffer flow velocity and sample concentration in the $3 \mathrm{D}$ device. The SIMPLEC algorithm was used for pressure-velocity coupling. The differencing schemes of upwind and second order limiter with blending factor of 0.1 are, respectively, used for discretization of the velocity fields and analyte distribution. The algebraic multigrid (AMG) iterative method was applied for solving the linearized algebraic equations for an accelerated convergence. The computational domain is meshed by a block-structured grid. To capture the steep concentration gradient generated at combining and splitting junctions and mitigate numerical diffusion errors [32], very fine meshes in the transverse direction are used. In addition, a polynomialtype mesh in the axial direction ( $x$-coordinate) is also selected to enable fine meshes at the junction and resolve flow entry effects on sample transport (inset in figure 5). To compare systematic modeling and numerical results, a scalar index is defined [20]:

$$
M=\int_{0}^{1}\left|c_{N}-c\right| \mathrm{d} \eta / \int_{0}^{1} c_{N} \mathrm{~d} \eta
$$

where $c$ is the normalized concentration profile from the analytical system models and $c_{N}$ is the depth-averaged concentration profile from numerical analysis. Hence, $M$ represents the error of systematic modeling results relative to the numerical analysis. The smaller $M$, the better the agreement.

\subsection{Experimental validation}

Our systematic modeling results are compared with experimental data reported by Walker et al [17], who investigated the evolution of an initial stepwise profile in the main mixing channel of the tree-like generator (figure 1). The transverse concentration profile at the main channel inlet consists of five discontinuous concentration steps arising from the serial splitting, combing and mixing of the sample in the tree-like network, and is quantitatively described 


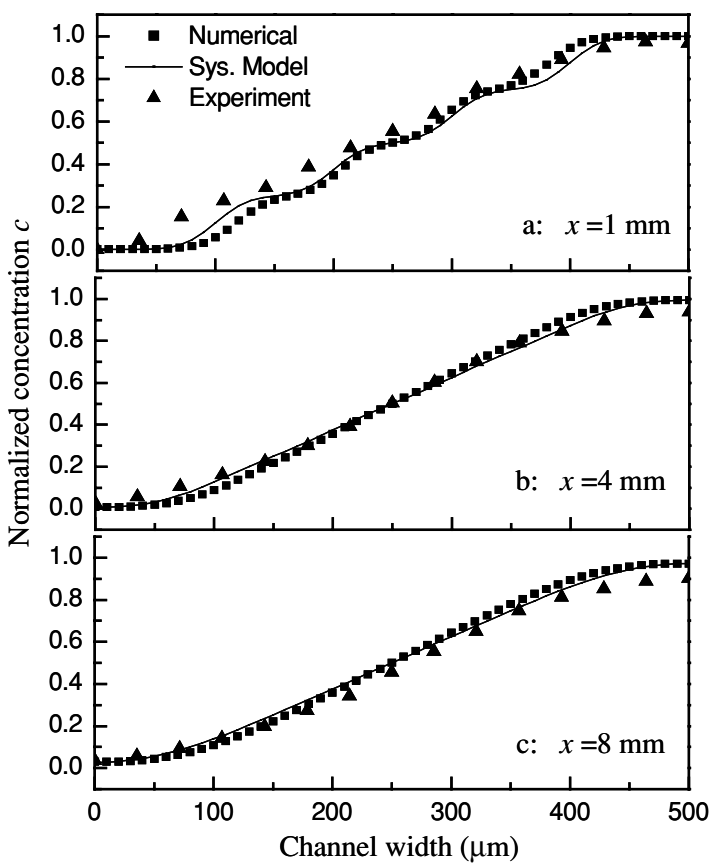

Figure 3. The systematic modeling results are compared with the numerical analysis and experimental data from the literature [17] on the evolution of an initial stepwise (five steps) profile in the main mixing channel of a tree-like gradient generator. (a) $1 \mathrm{~mm},(b) 4 \mathrm{~mm}$ and (c) $8 \mathrm{~mm}$ downstream of the channel inlet.

as: $\left.c\right|_{x=0}=0$ for $0 \leqslant \eta \leqslant 0.2 ;\left.c\right|_{x=0}=0.25$ for $0.2<\eta \leqslant 0.4$; $\left.c\right|_{x=0}=0.5$ for $0.4<\eta \leqslant 0.6 ;\left.c\right|_{x=0}=0.75$ for $0.6<\eta \leqslant 0.8$; $\left.c\right|_{x=0}=1$ for $0.8<\eta \leqslant 1$. Figure 3 shows the variation of the sample (FITC-dextran) concentration profile along the main mixing channel width ( $y$-coordinate) at three different axial positions ( $x$-coordinate), obtained from the system model, numerical analysis as well as extracted experimental data. It can be seen that the system model agrees well with the numerical and experimental data. The worst relative error is $M=4.6 \%$ at $x=1 \mathrm{~mm}$, which mainly occurs at the channel walls and can be attributed to the associated non-uniform transverse velocity profile as well as the non-fully developed concentration distributions along the channel depth. The discontinuity of the initial profile (at the channel inlet) smears out due to transverse diffusion as the sample migrates down the mixing channel. It is thus concluded that our analytical

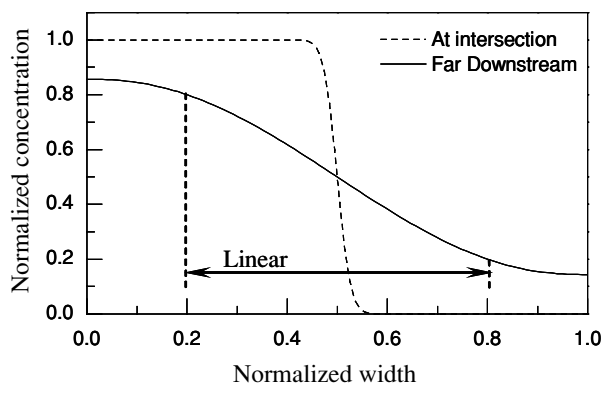

(a) system model has sufficient accuracy to be used to guide the gradient generator design.

\section{Application to concentration gradient generator design}

In this section, we will illustrate the utility of our systematic modeling approach by applying it to design of practically useful concentration gradient generators. Specifically, the approach is used to model a novel and compact generator that takes advantage of partial mixing. The modeling results again will be compared with the $3 \mathrm{D}$ numerical analysis. Note that while we choose to focus on devices that exploit partial mixing, those based on complete mixing can be treated as a straightforward special case.

Although complex concentration gradients can be generated from complete mixing [12, 15, 16], they can be implemented with more efficient and compact partial mixing devices, which however have not been adequately explored. Here we propose a generator device which consists of a simple collection of Y- (or T-) and cross-mixers whose incompletely mixed concentration profiles are combined to produce desired complex gradients. In a Y-mixer, sample and buffer solutions merge at the junction and then mix with each other in the downstream mixing channel. The extent of sample mixing shapes its concentration profiles (figure 4(a)), for example, immediately after the junction, an abrupt step-shaped profile results and the transverse position of the discontinuity is determined by sample and buffer flow rates. However at the far downstream, an approximately linear concentration profile forms, which exhibits a good linearity at the channel center ( $\sim 20-80 \%$ of the channel width). Similarly, a bell-shaped profile that has been employed in [2] to investigate neutrophil chemotaxis can be created by a cross-mixer (figure 4(b)). The sample is injected from the middle channel and sandwiched by buffer solvent from side channels [20]. Inter-stream diffusion in the mixing channel smoothes and produces sample concentration profiles with different width and height. By juxtaposing these constituent profiles, temporally and spatially stable gradients of complex shapes (figures 5 and 7) can be attained. As the constituent profiles are independent of each other, their shape characteristics (e.g., the width, slope, peak and mean concentration values) can be individually changed by choice of branch flow rates, channel sizes and reservoir

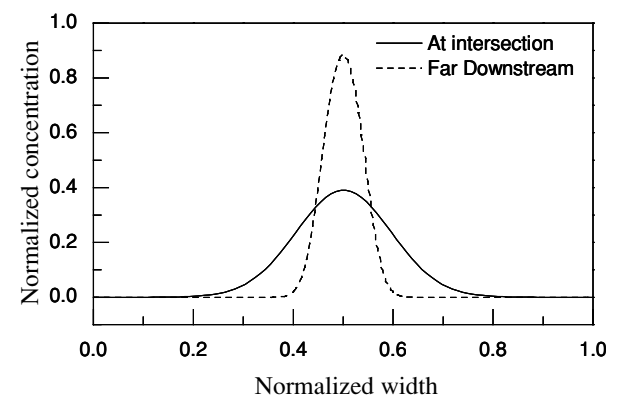

(b)

Figure 4. The transverse sample concentration profiles immediately after and far downstream of the merging junction in (a) a Y-mixer and (b) a cross-mixer. 


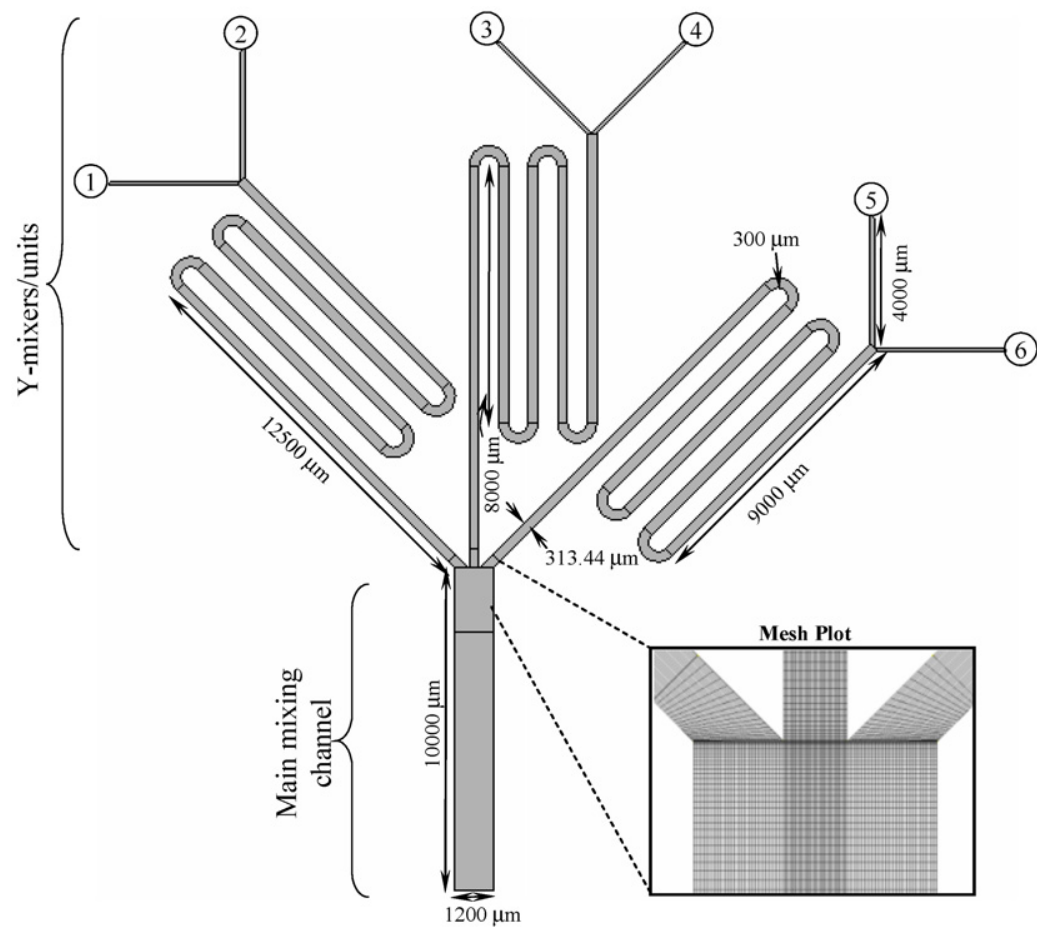

Figure 5. Network topology and dimensions of a saw-tooth concentration generator. A saw-tooth-shaped profile is produced by combining three constituent linear profiles, each evolving from a single Y-mixer. The inset shows the grid plot of the cross junction in the numerical (CFD-ACE+) simulations. 156 cells in the transverse direction of the main channel are used to resolve the steep gradient. Axially, more grid cells are used at the merging junction region to capture the flow entry effects on sample transport.

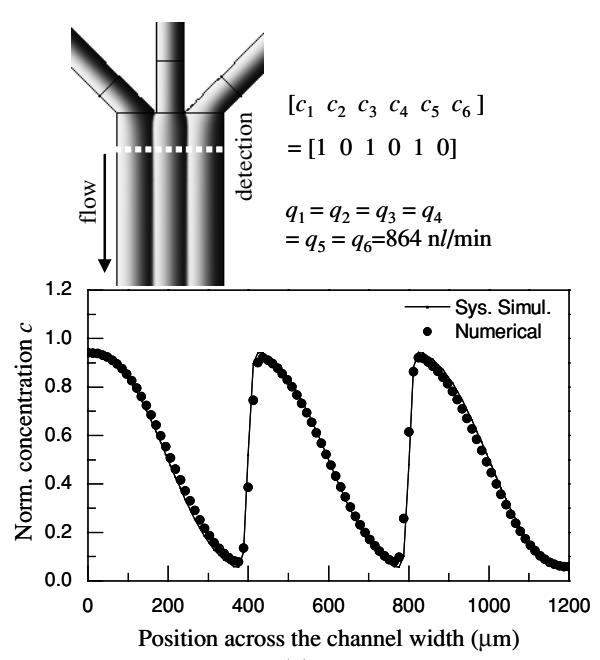

(a)

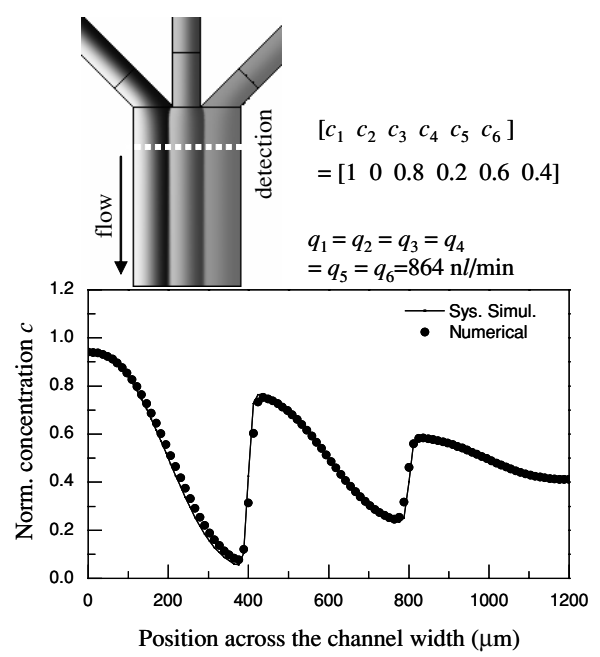

(b)

Figure 6. Numerical contour plots of the sample concentration and comparison between numerical analysis and systematic modeling results on concentration profiles across the channel width (extracted $400 \mu \mathrm{m}$ downstream of the junction). The flow rate in all branch channels $1-6$ is $864 \mathrm{nl} \mathrm{min} \mathrm{m}^{-1}$. The initial reservoir sample concentration values are shown above the individual profile. Three approximately linear constituent profiles with $(a)$ the same peak and mean concentration values and slope and $(b)$ the same mean values but different slopes and peak values.

sample concentrations. In the examples below, all channels have a depth of $h=60 \mu \mathrm{m}$ with an aspect ratio $\beta$ of 5-20. The volumetric flow rate in the main mixing channel (after juxtaposition of the constituent profiles) is fixed at $5.18 \mu 1 \mathrm{~min}^{-1}$, corresponding to a practically relevant velocity of $1200 \mu \mathrm{m} \mathrm{s}^{-1}$ [17]. We use a typical sample diffusivity of $D=1 \times 10^{-10} \mathrm{~m}^{2} \mathrm{~s}^{-1}$, and the normalized reservoir sample concentration ranging from 0 to 1 , unless otherwise noted.

\subsection{Saw-tooth-shaped concentration profiles}

Transverse saw-tooth-shaped concentration profiles [12] can be generated by juxtaposing multiple Y-mixing units (figure 5). Sample solutions with different concentrations are released from two adjacent reservoirs in a Y-mixing unit (e.g., reservoirs 1 and 2), and then mix with each other in the serpentine mixing channel to generate an approximately linear profile. The peak and mean concentration value and the slope of the constituent 


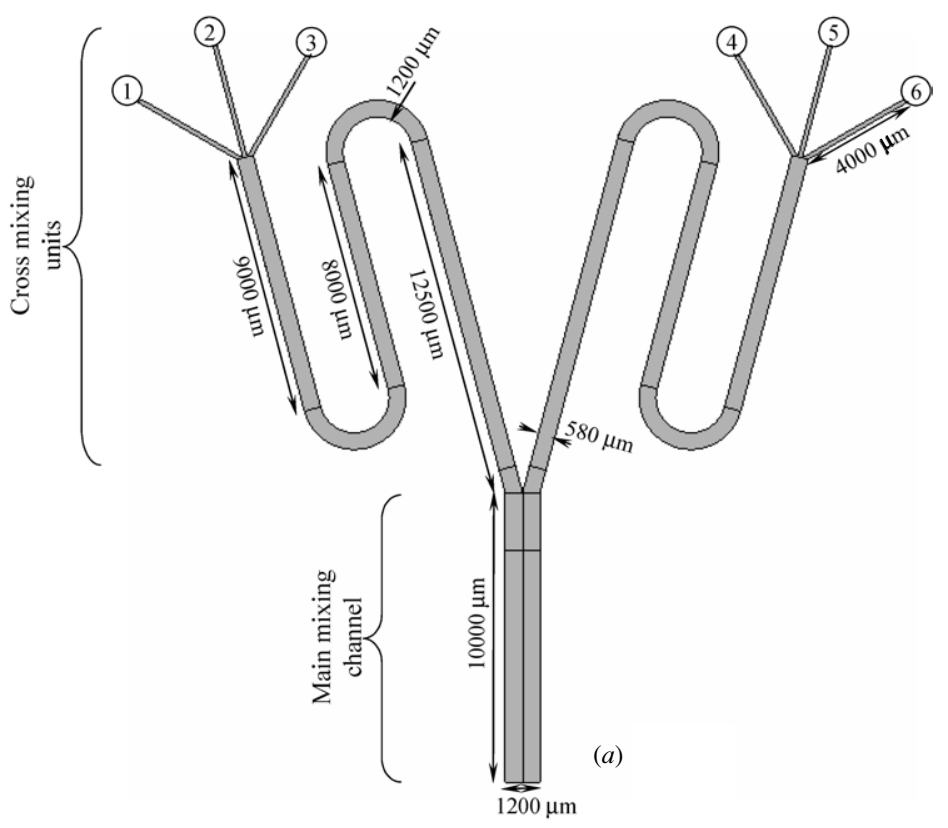

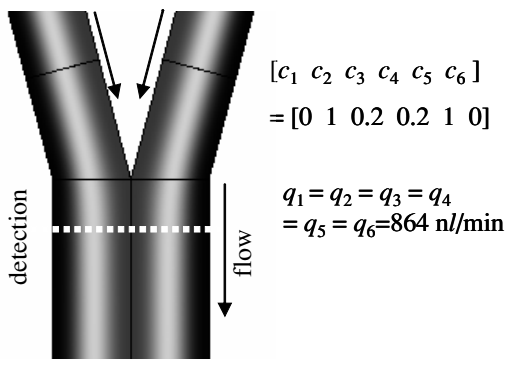

(b)

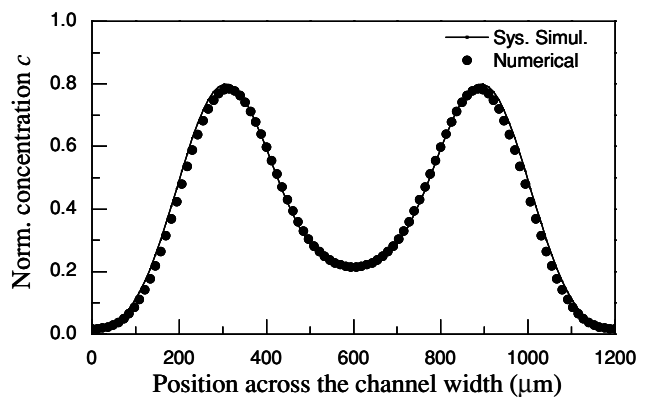

(c)

Figure 7. (a) Network topology and dimensions of a multiple-bell-shaped concentration generator. The profile is produced by combining two constituent bell-shaped profiles, each arising from a single cross-mixing unit. $(b)$ Numerical contour plot of the sample concentration at the merging junction. $(c)$ Comparison between numerical and systematic modeling results on the transverse concentration profile, extracted at $400 \mu \mathrm{m}$ downstream of the junction in the main mixing channel. Flow rate in branch channels $1-6$ is $864 \mathrm{nl} \mathrm{min}^{-1}$.

profile are determined from the initial reservoir concentrations, serpentine channel sizes, and sample flow rates that can be effectively captured by our system model (equations (5) and (7)). Eventually, the approximately linear profiles from serpentine mixing channels are combined in the main mixing channel to achieve a saw-tooth-shaped distribution.

Figure 6 shows various saw-tooth-shaped profiles from systematic and computational modeling results. The device in figure 5 has the highest Reynolds number $R e=0.15$ and Dean number $D e=0.07$, i.e., the effects of secondary flow in the turns are negligible (see section 3.2). Thus, we could treat the serpentine channel including multiple elements (straight channels and turns) as a single channel whose length is the sum of those of all elements, and further reduce computational costs. Specifically, six flow reservoirs, one waste reservoir, eleven channels and five combiners are used in the systematic simulation. The slopes, mean and peak values of each constituent profile are varied by modulating its corresponding reservoir sample concentrations. In figure $6(a)$, the exactly same linear profiles are duplicated along the channel width. Figure $6(b)$ demonstrates a saw-tooth shape consisting of three constituent concentration profiles, each having the same mean values but different peak values and slopes. As an extreme case, given a sufficiently fast flow velocity or an extremely short serpentine mixing channel, sample mixing in the Y-mixing unit is negligible, leading to a profile comprised of three square waves in the main channel. In figure 6, steep concentration discontinuities are created at the interface between streams, which can be useful for studying the cell behavior subjected to abrupt gradients [2]. Excellent agreement between numerical and systematic modeling results with the worst-case error of $M=4.8 \%$ indicates that our model is able to accurately predict discontinuous profiles as well as their evolution along the stream direction.

\subsection{Multiple-bell-shaped concentration profiles}

To create a multiple-bell-shaped concentration profile, crossmixing units can be assembled laterally (figure $7(a)$ ). Constituent bell-shaped concentration profiles first form in the cross-mixing units as described above, which then merge in the main mixing channel to create a multiple-bell-shaped 
profile. Figures $7(b)$ and $(c)$ show the numerical contour plot of the sample concentration as well as the comparison between numerical analysis and systematic modeling results. Different from that in figure $4(b)$, asymmetric constituent bell-shaped profiles are generated in the cross-mixing units, which is enabled by feeding different sample concentrations at reservoirs 1, 3 and 4, 6 (figure $7(b)$ ), leading to a higher sample concentration value at the centerline of the main channel. In the systematic simulation, six flow reservoirs, one waste reservoir, ten channels and five combiners are used for this example. An excellent agreement between numerical and systematic modeling results has been obtained with the relative error $M=4 \%$. Finally, we could also generate a hybrid concentration profile consisting of both linear and bell-shape profiles by combining cross- and Y-mixing units (results not shown). A good agreement between numerical analysis and system model with $M=4.9 \%$ is achieved.

The generator design enables accurate and efficient generation of complex gradients in a simple network, as long as the desired concentration profile does not include a strictly linear or parabolic shape. The impermeability of channel walls to mass transfer causes bending of the profile at the sidewalls as shown in figure 4, making strictly linear or parabolic gradients inaccessible. This limitation could be addressed by attaching three splitting channels at the end of the Y- or cross-mixing channel. Thus, the bent sides of the concentration profile are chopped off and the strictly linear or parabolic portion in the middle is remained and output for analysis. In this occasion, with inclusion of the splitter model (equations (9) and (10)), our systematic approach is still applicable.

Our system model has demonstrated vast improvements in computational efficiency over the numerical analysis. On a PC with an AMD Athlon $2 \mathrm{GHz}$ processor and $1 \mathrm{~GB}$ RAM operating in MS-Windows, the systematic simulations of the saw-tooth-shaped (with 100 Fourier terms) and multiple bellshaped gradient generators (with 10 Fourier terms) above were completed, respectively, within 5 and $1 \mathrm{~s}$ using $\sim 15$ MB RAM. These correspond to at least 3000-fold speed-up compared to numerical simulations, which used 4.7 and $4.3 \mathrm{~h}, 890000$ and 820000 grid cells, 990 and 930 MB memory, respectively, for the saw-tooth and bell-shaped profiles on the same platform. The computational advantage of our system model is thus clear, making possible systematic optimal design of gradient generators, which may involve hundreds or thousands of iterative simulations to achieve a user-specified concentration gradient.

\section{Conclusion}

A systematic modeling approach has been presented for use as a tool to guide the design of concentration gradient generators. A generator with virtually arbitrary geometry is represented as a collection of elements (e.g., mixing channels, combiners and splitters) with relatively simple geometries. Analytical and parameterized models for such elements in pressuredriven flow have been developed to capture the overall effects of generator geometry, material properties and operational procedures on sample transport. These element models are then integrated, by use of the appropriate interface parameters at element terminals, into a network, resulting in a system model for the entire generator. The model has been compared with experimental data extracted from the literature, as well as with the full numerical analysis.

The generator system model has been exploited to propose a novel generator capable of creating stable and complex concentration gradients. The fundamental approach is to juxtapose simple (e.g., approximately linear and bell-shaped) constituent concentration profiles resulting from Y- or crossmixers to obtain composite profiles with a higher level of complexity (e.g., saw-tooth and multiple-bell shapes). The class of achievable concentration profiles (e.g., linear or bellshaped) is determined from the type of the mixers (e.g., Y- or cross-mixers) and their spatial configurations. The constituent profile details (e.g., slope, mean and peak concentration values, position and width) are primarily determined from the initial reservoir concentrations, flow rates, sample properties as well as the channel dimensions. Therefore, upon integrating with optimization algorithm and iterative evaluation engines, our system model can be used to guide the selection of appropriate parameters to attain desired concentration profiles.

\section{Acknowledgments}

This research is sponsored by DARPA and the Air Force Research Laboratory, Air Force Material Command, USAF, under grant number F30602-01-2-0587, and the NSF ITR program under award number CCR-0325344.

\section{References}

[1] Wang S J, Saadi W, Lin F, Nguyen C M C and Jeon N L 2004 Differential effects of EGF gradient profiles on MDA-MB-231 breast cancer cell chemotaxis Exp. Cell Res. 300 180-9

[2] Jeon N L, Baskaran H, Dertinger S K W, Whitesides G M, Van de Water L and Toner M 2002 Neutrophil chemotaxis in linear and complex gradients of interleukin-8 formed in a microfabricated device Nature Biotechnol. 20 826-30

[3] Jiang X Y, Ng J M K, Stroock A D, Dertinger S K W and Whitesides G M 2003 A miniaturized, parallel, serially diluted immunoassay for analyzing multiple antigens J. Am. Chem. Soc. 125 5294-5

[4] Mao H B, Yang T L and Cremer P S 2002 Design and characterization of immobilized enzymes in microfluidic systems Anal. Chem. 74 379-85

[5] Jiang X Y, Xu Q B, Dertinger S K W, Stroock A D, Fu T M and Whitesides G M 2005 A general method for patterning gradients of biomolecules on surfaces using microfluidic networks Anal. Chem. 77 2338-47

[6] Kenis P J A, Ismagilov R F, Takayama S, Whitesides G M, Li S L and White H S 2000 Fabrication inside microchannels using fluid flow Accounts Chem. Res. 33 841-7

[7] Boyden S 1962 Chemotactic effect of mixtures of antibody and antigen on polymorphonuclear leucocytes J. Exp. Med. $115453-66$

[8] Weiner O D, Servant G, Welch M D, Mitchison T J, Sedat J W and Bourne H R 1999 Spatial control of actin polymerization during neutrophil chemotaxis Nature Cell Biol. 1 75-81

[9] Wilkinson P C and Lackie J M 1983 The influence of contact guidance on chemotaxis of human neutrophil leukocytes Exp. Cell Res. 145 255-64

[10] Zicha D, Dunn G A and Brown A F 1991 A new directviewing chemotaxis chamber J. Cell Sci. 99 769-75 
[11] Zigmond S H 1977 Ability of polymorphonuclear leukocytes to orient in gradients of chemotactic factors J. Cell Biol. 75 606-16

[12] Dertinger S K W, Chiu D T, Jeon N L and Whitesides G M 2001 Generation of gradients having complex shapes using microfluidic networks Anal. Chem. 73 1240-6

[13] Holden M A, Kumar S, Castellana E T, Beskok A and Cremer P S 2003 Generating fixed concentration arrays in a microfluidic device Sensors Actuators B 92 199-207

[14] Jacobson S C, McKnight T E and Ramsey J M 1999 Microfluidic devices for electrokinetically driven parallel and serial mixing Anal. Chem. 71 4455-9

[15] Jeon N L, Dertinger S K W, Chiu D T, Choi I S, Stroock A D and Whitesides G M 2000 Generation of solution and surface gradients using microfluidic systems Langmuir $168311-6$

[16] Lin F, Saadi W, Rhee S W, Wang S J, Mittal S and Jeon N L 2004 Generation of dynamic temporal and spatial concentration gradients using microfluidic devices Lab on a Chip 4 164-7

[17] Walker G M, Sai J Q, Richmond A, Stremler M, Chung C Y and Wikswo J P 2005 Effects of flow and diffusion on chemotaxis studies in a microfabricated gradient generator Lab on a Chip 5 611-8

[18] Walker G M, Ozers M S and Beebe D J 2004 Cell infection within a microfluidic device using virus gradients Sensors Actuators B 98 347-55

[19] Biddiss E and Li D Q 2005 Electrokinetic generation of temporally and spatially stable concentration gradients in microchannels J. Colloid Interface Sci. 288 606-15

[20] Wang Y, Lin Q and Mukherjee T 2005 A model for laminar diffusion-based complex electrokinetic passive micromixers Lab on a Chip 5 877-87
[21] Wang Y, Lin Q and Mukherjee T 2006 Composable behavioral models and schematic-based simulation of electrokinetic lab-on-a-chip systems IEEE Trans. Comput.-Aided Des. Integr. Circuits Syst. 25 258-73

[22] White F M 1991 Viscous Fluid Flow 2nd edn (New York: McGraw-Hill)

[23] Coelho C P, Desai S D, Freeman D and White J 2005 A robust approach for estimating diffusion constants from concentration data in microchannel mixers Proc. 2005 NSTI Nanotechnology Conf. and Trade Show (Nanotech 2004) (Anaheim, CA, 2005)

[24] Kamholz A E, Weigl B H, Finlayson B A and Yager P 1999 Quantitative analysis of molecular interaction in a microfluidic channel: the T-sensor Anal. Chem. 71 5340-7

[25] Bothe D, Sternich C and Warnecke H J 2006 Fluid mixing in a T-shaped micro-mixer Chem. Eng. Sci. 61 2950-8

[26] Schilling E A, Kamholz A E and Yager P 2002 Cell lysis and protein extraction in a microfluidic device with detection by a fluorogenic enzyme assay Anal. Chem. 74 1798-804

[27] Jiang F, Drese K S, Hardt S, Kupper M and Schönfeld F 2004 Helical flows and chaotic mixing in curved micro channels AIChE J. 50 2297-305

[28] Schönfeld F and Hardt S 2004 Simulation of helical flows in microchannels AIChE J. 50 771-8

[29] Vanka S P, Luo G and Winkler C M 2004 Numerical study of scalar mixing in curved channels at low Reynolds numbers AIChE J. 50 2359-68

[30] http://www.wolfram.com

[31] ESI-CFD Inc. 2004 CFD-ACE+ Theory Manual

[32] Hardt S and Schönfeld F 2003 Laminar mixing in different interdigital micromixers: II. Numerical simulations $A I C h E$ J. $49578-84$ 